\title{
Dust emissivity in the star-forming filament OMC 2/3 ${ }^{\star}$
}

\author{
S. I. Sadavoy ${ }^{1}$, A. M. Stutz ${ }^{1}$, S. Schnee ${ }^{2}$, B. S. Mason ${ }^{2}$, J. Di Francesco ${ }^{3,4}$, and R. K. Friesen ${ }^{5}$ \\ ${ }^{1}$ Max-Planck-Institut für Astronomie (MPIA), Königstuhl 17, 69117 Heidelberg, Germany \\ e-mail: sadavoy@mpia-hd.mpg.de \\ 2 National Radio Astronomy Observatory, 520 Edgemont Road, Charlottesville, VA 22903, USA \\ 3 National Research Council Canada, 5071 West Saanich Road Victoria, BC V9E 2E7, Canada \\ ${ }^{4}$ Department of Physics \& Astronomy, University of Victoria, PO Box 3055 STN CSC, Victoria, BC V8W 3P6, Canada \\ 5 Dunlap Institute for Astronomy and Astrophysics, University of Toronto, 50 St. George Street, Toronto M5S 3H4, Ontario, Canada
}

Received 14 September 2015 / Accepted 22 January 2016

\begin{abstract}
We present new measurements of the dust emissivity index, $\beta$, for the high-mass, star-forming OMC $2 / 3$ filament. We combined $160-500 \mu \mathrm{m}$ data from Herschel with long-wavelength observations at $2 \mathrm{~mm}$ and fit the spectral energy distributions across a $\simeq 2 \mathrm{pc}$ long, continuous section of OMC $2 / 3$ at $15000 \mathrm{AU}(0.08 \mathrm{pc})$ resolution. With these data, we measured $\beta$ and reconstructed simultaneously the filtered-out large-scale emission at $2 \mathrm{~mm}$. We implemented both variable and fixed values of $\beta$, finding that $\beta=1.7-1.8$ provides the best fit across most of OMC 2/3. These $\beta$ values are consistent with a similar analysis carried out with filtered Herschel data. Thus, we show that $\beta$ values derived from spatial filtered emission maps agree well with those values from unfiltered data at the same resolution. Our results contradict the very low $\beta$ values $(\sim 0.9)$ previously measured in OMC $2 / 3$ between $1.2 \mathrm{~mm}$ and $3.3 \mathrm{~mm}$ data, which we attribute to elevated fluxes in the $3.3 \mathrm{~mm}$ observations. Therefore, we find no evidence of rapid, extensive dust grain growth in OMC 2/3. Future studies with Herschel data and complementary ground-based long-wavelength data can apply our technique to obtain reliable determinations of $\beta$ in nearby cold molecular clouds.
\end{abstract}

Key words. stars: formation - dust, extinction - ISM: clouds - submillimeter: ISM

\section{Introduction}

Dust grains are excellent tracers of mass and structure in molecular clouds. Thermal emission from dust can characterize structures over various scales, from the diffuse cloud to the dense, star-forming cores (e.g., Di Francesco et al. 2008; Enoch et al. 2009; André et al. 2010; Stutz \& Kainulainen 2015). Nevertheless, the conversion of thermal dust emission to mass is nontrivial, mainly because of uncertain dust emissivities. Most studies assume a single power-law distribution for the dust emissivity, $v^{\beta}$, resulting in masses that are uncertain by factors of a few (e.g., Henning et al. 1995; Shirley et al. 2011).

The dust emissivity index, $\beta$, represents the efficiency at which dust grains radiate at long wavelengths, where the value $\beta=2$ is expected for bare dust grains in the interstellar medium (e.g., Draine \& Lee 1984). This efficiency, however, will evolve with density and temperature. For example, dust grains in cold, dense cores are likely to coagulate, (e.g., Ossenkopf \& Henning 1994; Ormel et al. 2011), leading to values of $\beta<2$ toward molecular clouds and dense cores (e.g., Shirley et al. 2011; Planck Collaboration XXIV 2011; Sadavoy et al. 2013) and values of $\beta<1$ toward protoplanetary disks (e.g., Beckwith \& Sargent 1991; Wright et al. 2015).

The Orion molecular cloud (OMC) $2 / 3$ region is an active, star-forming filament with a rich population of young stars

* A FITS image for the GISMO $2 \mathrm{~mm}$ observations is only available at the CDS via anonymous ftp to

cdsarc.u-strasbg.fr (130.79.128.5) or via

http://cdsarc.u-strasbg.fr/viz-bin/qcat?]/A+A/588/A30
(Peterson \& Megeath 2008). OMC 2/3 also represents one of the closest sites of high-mass star formation ( $420 \mathrm{pc}$; Menten et al. 2007), making this region an interesting target to compare with nearby low-mass molecular clouds. Indeed, many previous studies have examined its dust properties including the dust emissivity index, $\beta$. Chini et al. (1997) used data between 350-2000 $\mu \mathrm{m}$ to determine $\beta$ toward nine cores in OMC $2 / 3$. For a reasonable range of assumed temperatures, Chini et al. (1997) found that most of their sources were well-fit with $\beta=2$, although several had best-fit values of $\beta \lesssim 1.5$. Similarly, Johnstone \& Bally (1999) used SCUBA $450 \mu \mathrm{m}$ and $850 \mu \mathrm{m}$ ratios to characterize $\beta$ across the entire Orion integral shaped filament. For $T>10 \mathrm{~K}$, their data agreed well with $1<\beta<2$, although their results could not rule out lower $\beta$ indices at higher temperatures.

While these earlier studies found $\beta$ values that agreed well with theoretical predictions for dust grains within molecular clouds and cores, a recent study by Schnee et al. (2014) found much lower $\beta$ values. Schnee et al. (2014) used ratios of $1.2 \mathrm{~mm}$ and $3.3 \mathrm{~mm}$ observations with $\mathrm{NH}_{3}$-derived temperatures (from Li et al. 2013) to determine $\beta$ in OMC $2 / 3$ for scales up to $\sim 0.1 \mathrm{pc}$. They found $\beta=0-2$ throughout the OMC $2 / 3$ filament, with a median value of $\beta \simeq 0.9$ and values of $\beta \lesssim 1$ toward most of the dense cores. These $\beta$ indices are considerably lower than earlier measurements and are usually associated with millimeter-size dust grains that are primarily found in disks (e.g., Beckwith \& Sargent 1991; Williams \& Cieza 2011) and not with the micron-size dust grains associated with filaments and cores (e.g., Pagani et al. 2010; Testi et al. 2014). These results suggest that extraordinary processes may be occurring in OMC $2 / 3$ 
that produce significant, wide-spread grain growth on $\sim 0.1 \mathrm{pc}$ scales. Evidence for such rapid grain evolution has not been before detected.

To explore the possibility of rapid dust grain growth in OMC 2/3, we combined 160-500 $\mu \mathrm{m}$ observations from the Herschel Space Observatory with $2 \mathrm{~mm}$ observations from the IRAM $30 \mathrm{~m}$ telescope to determine $\beta$ on larger scales than in Schnee et al. (2014). The $2 \mathrm{~mm}$ data are necessary to constrain fits to spectral energy distributions (SEDs) because of the degeneracy between temperature and $\beta$ (Shetty et al. 2009b; Ysard et al. 2012; Juvela et al. 2013). With the Herschel and $2 \mathrm{~mm}$ observations, we obtained an independent measure of $\beta$ in OMC $2 / 3$.

\section{Data}

\subsection{Herschel}

The Orion molecular cloud was observed as part of the Herschel Gould Belt Survey (André et al. 2010). The cloud was mapped in the PACS/SPIRE parallel mode to observe the cloud simultaneously at $70 \mu \mathrm{m}, 160 \mu \mathrm{m}, 250 \mu \mathrm{m}, 350 \mu \mathrm{m}$, and $500 \mu \mathrm{m}$. We used the 160-500 $\mu \mathrm{m}$ maps presented in Stutz \& Kainulainen (2015) and Stutz \& Gould (2015), which were made by processing the Level 1 map products of the Herschel Science Archive with scanamorphos (version 24.0, Roussel 2013). The Herschel data were corrected for zero-point fluxes using IRAS and Planck data (e.g., following Bernard et al. 2010).

\subsection{IRAM $30 \mathrm{~m}$ telescope}

The OMC 2/3 complex was observed at $2 \mathrm{~mm}$ with GISMO (Staguhn et al. 2008) at the IRAM $30 \mathrm{~m}$ telescope on 5 April 2014 in good weather $\left(\tau_{225 \mathrm{GHz}} \lesssim 0.3\right)$. We used orthogonal $\sim 7^{\prime}$ scans at angles of $\pm 30^{\circ}$ to produce a final map of $\sim 7^{\prime} \times 22^{\prime}$ at a $40 \operatorname{arcsec~s}^{-1}$ scan rate. The total on-sky time was $\sim 1 \mathrm{~h}$. A larger map $\left(\sim 8^{\prime} \times 34^{\prime}\right)$ that includes the OMC 1 region was observed in the same manner on 10 April 2014 in poor weather conditions $\left(\tau_{225 \mathrm{GHz}} \sim 0.5\right)$ for an on-sky time of $\sim 1 \mathrm{~h}$. Additional observations taken at low elevation $\left(<30^{\circ}\right)$ and high airmass on 9 April 2014 were not used in this analysis.

The scans of 5 and 10 April were reduced together using CRUSH (Kovács 2008) vers. 2.22 with the "faint" and "extended" modes and extra iterations. With these parameters, we expect to recover emission on $\sim 2^{\prime}$ scales to $90 \%$ (A. Kovács 2015, priv. comm.; see also Appendix A). For the OMC 2/3 data, we obtain an $1 \sigma$ rms sensitivity of $\sim 2.5$ mJy beam $^{-1}$ for a $\sim 21^{\prime \prime}$ FWHM beam. For the OMC 1 complex, which was only observed on 10 April, the $1 \sigma \mathrm{rms}$ sensitivity is $\sim 4 \mathrm{mJy}^{\text {beam }}{ }^{-1}$. We use only the OMC $2 / 3$ data at $2 \mathrm{~mm}$ hereafter.

\section{Results}

Figure 1 compares the Herschel-derived column density map of OMC 2/3 from Stutz \& Kainulainen (2015) with the $2 \mathrm{~mm}$ observations from GISMO. The column densities were determined from SED fits to Herschel data alone, assuming a fixed dust opacity law based on the models of Ossenkopf \& Henning (1994). In general, the two maps agree well, suggesting that the Herschel-derived column densities trace the locations of dense material well. The $\mathrm{H}$ II region M 43, however, is a notable exception where bright $(>3 \sigma)$, extended emission at $2 \mathrm{~mm}$ does not correspond to an increase in column density from the Herschel

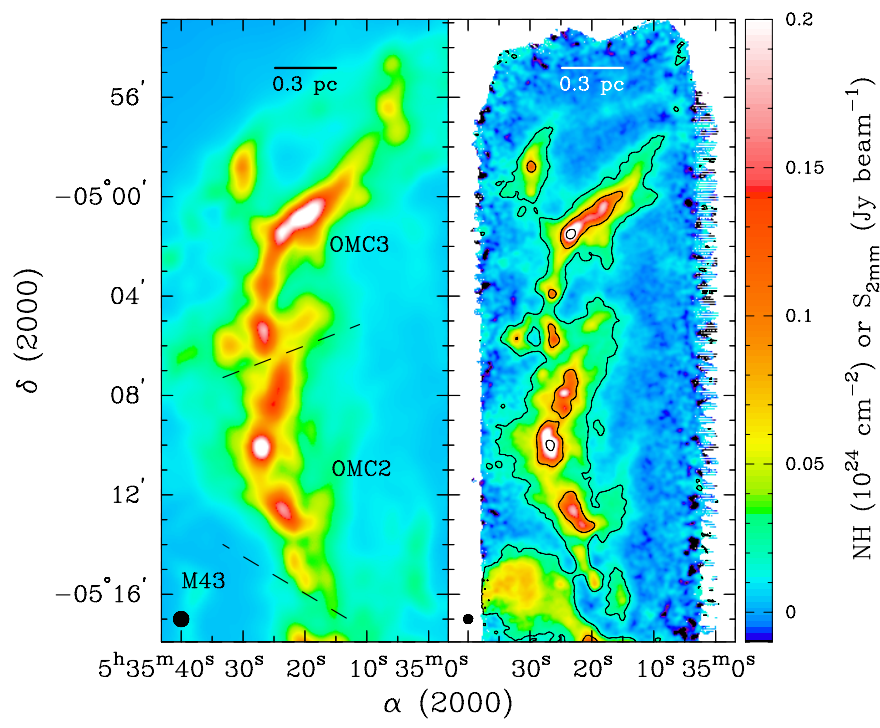

Fig. 1. Observations of OMC 2/3. Left: column densities from Stutz \& Kainulainen (2015) at $\sim 36^{\prime \prime}$ resolution and assuming a fixed dust opacity law. The complexes are separated by dashed lines based on Chini et al. (1997). The H II region, M 43, is also labeled. Right: IRAM 2 mm observations at $21^{\prime \prime}$ resolution. Contours show $2 \mathrm{~mm}$ flux density levels of $0.02,0.08,0.3$, and $1 \mathrm{Jy}^{\text {beam }}{ }^{-1}$. Respective beams are shown in the the lower left corners.

data. The $2 \mathrm{~mm}$ continuum is likely enhanced by free-free emission from the $\mathrm{H}$ II region. We exclude $\mathrm{M} 43$ from further analysis in this study.

For the OMC 2/3 filament, we convolved the Herschel and $2 \mathrm{~mm}$ data to a common resolution of 36 '. 3 and a common grid of $14^{\prime \prime}$ pixels, corresponding to the $500 \mu \mathrm{m}$ data. Dust temperatures and $\beta$ indices were determined from fitting the observed SEDs with the modified blackbody function,

$I_{v}=\kappa_{0}\left(v / v_{0}\right)^{\beta} B_{v}(T) \Sigma$,

where $\kappa_{0}$ is the dust opacity at the reference frequency $v_{0}, B_{v}$ is the blackbody function at temperature $T$, and $\Sigma$ is the gas mass column density. For this study, we assumed $\kappa_{0}=0.1 \mathrm{~cm}^{2} \mathrm{~g}^{-1}$ and $v_{0}=1 \mathrm{THz}$ in agreement with the Herschel Gould Belt Survey (André et al. 2010).

The SED fitting is nontrivial. Since the $2 \mathrm{~mm}$ observations were obtained at a ground-based facility, these data are filtered on scales larger than a few arcmin, whereas the Herschel data recover emission on these scales. Thus, the pixel-by-pixel SEDs will be missing emission at $2 \mathrm{~mm}$ relative to the Herschel data. To fit the Herschel $+2 \mathrm{~mm}$ SEDs, we must either recover the large-scale diffuse emission at $2 \mathrm{~mm}$ or similarly filter out the large-scale emission from the Herschel data. Here, we examine both possibilities.

\subsection{Unfiltered data}

In our first approach, we recovered the large-scale structure at $2 \mathrm{~mm}$ using a grid of offsets to represent the filtered-out largescale emission. For each assumed offset value, we fitted SEDs over subregions of $9 \times 9$ pixels $\left(2.1^{\prime} \times 2.1^{\prime}\right)$ where we expect the extended emission to be smooth (see Appendix A). We then identified the best-fit offsets for various assumed values of $\beta$. Offsets that deviate significantly from the true value of the extended emission will be poorly fit with a modified blackbody function to the 160-2000 $\mu \mathrm{m}$ SED, whereas those offset values 


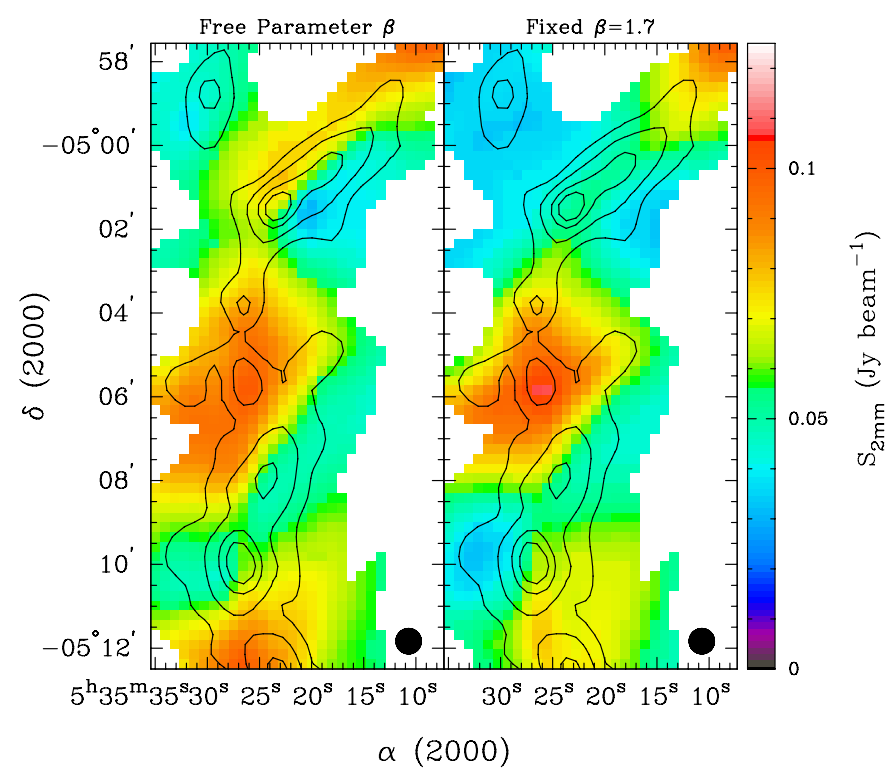

Fig. 2. Recovered large-scale emission ("offsets") for $\beta$ is a free parameter (left) and when $\beta=1.7$ (right). Contours show flux densities of $0.08,0.16,0.32$, and $0.48 \mathrm{Jy} \mathrm{beam}^{-1}$ from the observed $2 \mathrm{~mm}$ map at $36^{\prime \prime}$ resolution (black circle). Note that the structures in the offset map have scales $>5$ arcmin.

that match the true value of the extended emission well will produce better fits. By minimizing the global $\chi^{2}$ across all pixels in a $9 \times 9$ pixel subregion, we estimated the filtered-out large-scale emission toward each pixel. For more details, see Appendix D in Sadavoy et al. (2013).

We applied our technique with $\beta$ as a free parameter and with fixed values of $\beta=1.3-2.1$. For the $\beta$ trials, we adopted a grid of offsets from $0-0.2 \mathrm{Jy}^{\text {beam }^{-1}}$ for $\beta>1.4$ and a coarser grid of $0-0.4 \mathrm{Jy} \mathrm{beam}^{-1}$ for lower values of $\beta$. Moreover, we fitted only those pixels with $S_{2 \mathrm{~mm}}>5 \sigma$ to ensure that the observed $2 \mathrm{~mm}$ emission can be used as a constraint in the SED fit.

Each $\beta$ trial resulted in different best-fit offsets, $S_{\text {off }}$. For example, $\beta=2.1$ produced only best-fit offsets of $S_{\text {off }} \lesssim$ $0.03 \mathrm{Jy}^{\text {beam }^{-1}}$ throughout OMC $2 / 3$, whereas $\beta=1.3$ gave only best-fit offsets of $S_{\text {off }}>0.06 \mathrm{Jy}_{\text {beam }}{ }^{-1}$. Nevertheless, we see common features in most cases. Figure 2 shows the best-fit offset results for the cases of $\beta$ as a free parameter and $\beta=1$.7. In both maps, we find that $S_{\text {off }} \sim 0.3-0.1 \mathrm{Jy}$ beam $^{-1}$ across OMC $2 / 3$ with typical uncertainties of $\lesssim 20 \%$. Both maps also give similar, large-scale clumpy structures. These features are seen in many of the $\beta$ trials and most likely reflect the missing large-scale structure toward OMC $2 / 3$ at $2 \mathrm{~mm}$.

We identified the best-fit offsets for each $\beta$ trial, but not all $\beta$ trials produced reliable best-fit results. Figure 3 compares the best-fit results from several $\beta$ trials. The histograms show the minimum total $\chi^{2}$ value $\left(\chi_{\min }^{2}\right)$ for each pixel in each $\beta$ trial normalized by the median lowest $\chi^{2}$ value $\left(\widetilde{\chi}_{\min }^{2}\right)$ for that pixel across all presented trials. With this scaling, values $<1$ performed better than the median and values $>1$ performed worse. We find that $\beta \gtrsim 2$ and $\beta \lesssim 1.5$ do not fit our observations well, whereas $\beta=1.8$ and $\beta=1.7$ generally performed much better than the median value. The $2 \mathrm{~mm}$ band is very sensitive to slight changes in $\beta$. For example, a change from $\beta=2$ to $\beta=1.8$ at $20 \mathrm{~K}$ increases the $2 \mathrm{~mm}$ emission by $46 \%$, whereas the Herschel bands differ by $\lesssim 10 \%$.

Figure 4 shows the distribution of $\beta$ across OMC 2/3 from the trial with $\beta$ as a free parameter. We find a median value

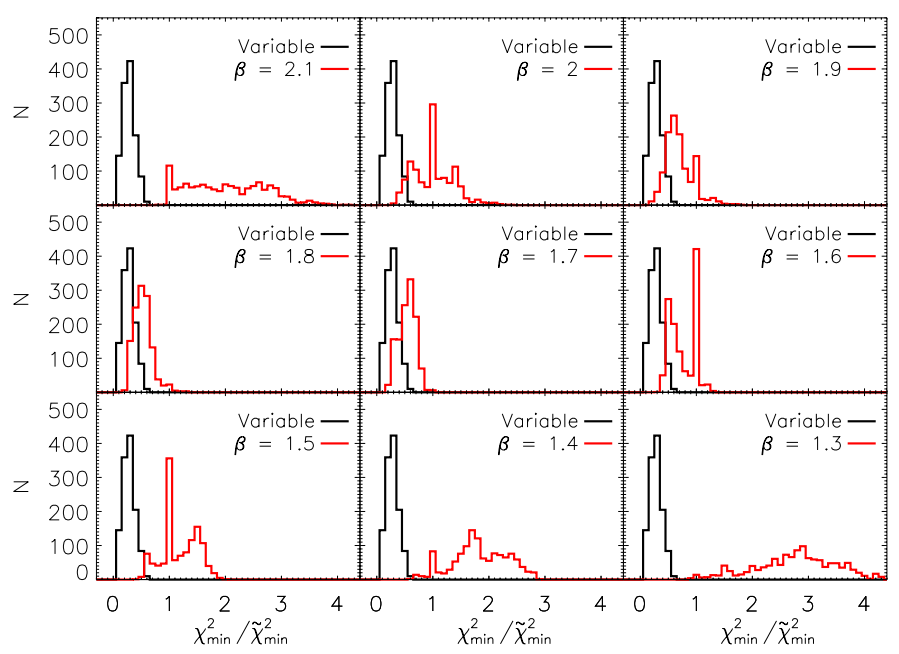

Fig. 3. Minimum $\chi^{2}$ values $\left(\chi_{\min }^{2}\right)$ from fits to each pixel scaled by the median lowest $\chi^{2}$ value $\widetilde{\chi}_{\min }^{2}$ ) from all ten trials for that pixel. The plots compare fixed values of $\beta$ (red histograms) with the case of $\beta$ as a free parameter (black histograms).

of $\beta=1.7$, in agreement with the fixed $\beta$ results (Fig. 3). Nevertheless, Fig. 4 shows $\beta \simeq 1.3$ toward the map edges and $\beta \simeq 1.4$ for MMS 6 . Along the map edges, the dust temperatures were $>25 \mathrm{~K}$ (see Sect. 4.5), and at these temperatures, SEDs are not constrained well with a shortest wavelength of $160 \mu \mathrm{m}$. The MMS 6 source has $T \simeq 20 \mathrm{~K}$, however, so its SED fits are still expected to be reliable. Assuming offset uncertainties of $20 \%$ and calibration uncertainties of $10 \%$ in each band (with the SPIRE calibration errors correlated), we conducted a Monte Carlo analysis of the measurement uncertainties in $\beta$. We found $\sigma_{\beta} \simeq 0.1$, indicating that the lower $\beta$ values toward MMS 6 are significant within the measurement uncertainties.

\subsection{Filtered data}

In our second approach, we filtered the Herschel data to match the spatial scales recovered in the GISMO $2 \mathrm{~mm}$ data. We adopted a similar Fourier-based technique as Wang et al. (2015) to remove the large-scale structures from our Herschel data. Similar to a highpass filter, we created a frequency domain filtering mask by fitting an exponential function to the $2 \mathrm{~mm}$ amplitude profile. This mask was designed to retain the smallscale structures and suppress the large-scale emission in a similar manner as the GISMO data. We applied this mask to the Herschel data in the frequency domain to produce our filtered Herschel maps. See Appendix A for more details.

We fitted the filtered Herschel+GISMO SEDs directly using Eq. (1). We processed only those pixels with $>5 \sigma$ in all wavebands to avoid artifacts in the filtering (see Appendix A). Figure 5 shows the distribution of $\beta$ in OMC $2 / 3$ from the filtered data. The filtered data produced a wider range in $\beta$ values than the unfiltered data, with $\beta=0.7-2.1$ across OMC $2 / 3$. The locations with $\beta \lesssim 1$, however, are exclusively toward the edges of the filament and most likely result from the filtering process (see Appendix A). Excluding these data points, the filtered data give a median value of $\beta \simeq 1$. 6 . Figure 5 also shows the ratio of the $\beta$ values obtained from the filtered and unfiltered data. Except for the filament edges, where we have possible filtering artifacts, the two datasets agree well. In general, we find that the filtered and unfiltered $\beta$ indices agree within $\sim 20 \%$, similar to 


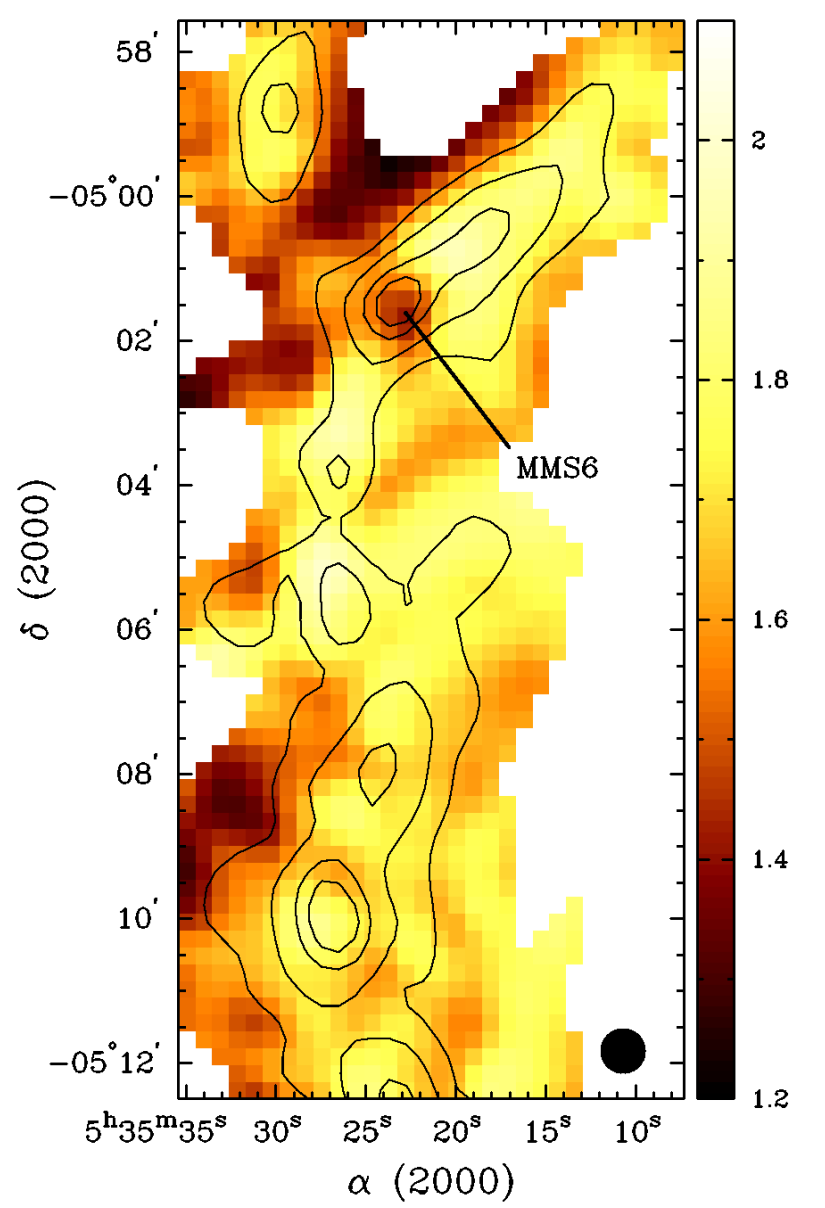

Fig. 4. Dust emissivity map of OMC $2 / 3$ when $\beta$ is a free parameter. Contours show observed $2 \mathrm{~mm}$ flux densities of $0.08,0.16,0.32$, and $0.48 \mathrm{Jy}^{-1}$ beam ${ }^{-1}$ at $36^{\prime \prime}$ resolution (black circle). The source MMS 6 (Chini et al. 1997) is also highlighted.

the measurement uncertainties, with lower indices (by a factor of $\gtrsim 0.7$ ) obtained from the filtered data.

Similar to the unfiltered case (Sect. 3.1) there are also hints of lower $\beta$ indices toward the MMS 6 source. Figure 5 covers MMS 6 only partially because this source was coincident with filtering artifacts, but there is sufficient structure away from these artifacts to suggest that $\beta \simeq 1.2$ toward MMS 6 . Thus, both methods suggest unique dust emissivities toward this one source.

\section{Discussion}

We found values of $\beta=1.7-1.8$ with our unfiltered analysis and $\beta \simeq 1.6$ with our filtered analysis across the OMC $2 / 3$ filament. The two methods agree within their uncertainties, suggesting that spatially filtering our observations did not greatly affect the derived values of $\beta$. We also found that the $\beta$ indices toward the MMS 6 core were consistently lower than those found along the main filament. MMS 6 has $\beta \simeq 1.4$ and $\beta \simeq 1.2$ with the unfiltered and filtered data, respectively. These unique values suggest that MMS 6 may have unique dust properties (see also, Sect. 4.2).

Our $\beta$ distributions agree well with the results from Chini et al. (1997) and Johnstone \& Bally (1999). Those studies similarly found $1<\beta<2$ for assumed temperatures typical of starforming regions. In particular, Chini et al. (1997) also found evidence of lower $\beta$ indices $(\beta \lesssim 1.5)$ toward the MMS 6 source, in agreement with our results (see also, Lis et al. 1998). In

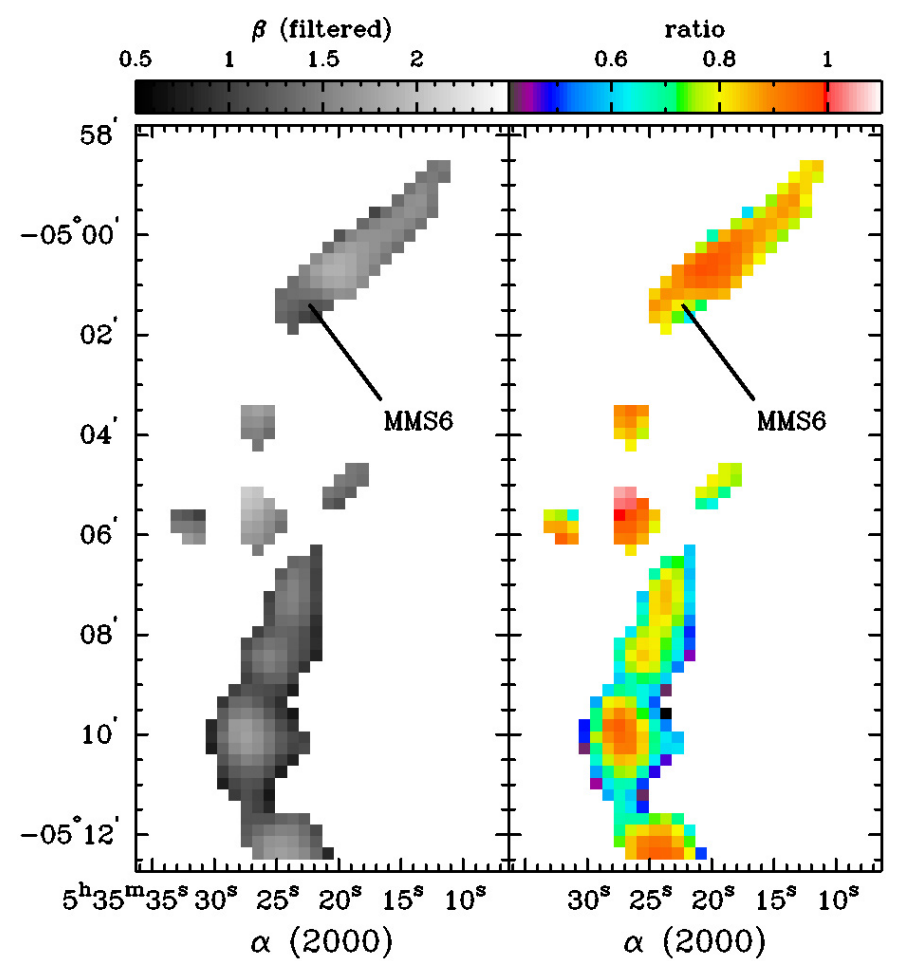

Fig. 5. Best-fit $\beta$ values from Fourier-filtered Herschel data and our observed GISMO $2 \mathrm{~mm}$ observations (left) and the ratio of these $\beta$ values to the $\beta$ values from the unfiltered data in Sect. 3.1.

comparison, Schnee et al. (2014) found much lower $\beta$ values compared to the results in this study and in the earlier studies of Chini et al. (1997) and Johnstone \& Bally (1999). For example, they found a median value of $\beta \simeq 0.9$ and values of $\beta \simeq 0.5$ toward MMS 6. Thus, the very low $\beta$ values from Schnee et al. (2014) appear to be an outlier. We investigate the reason for this $\beta$ discrepancy in the following sections.

\subsection{Dust emissivity in dense cores}

As an additional test of $\beta$ in OMC $2 / 3$, we examined the compact, dense cores themselves. We extracted source fluxes from the Herschel+GISMO data using the extraction code getsources (Men'shchikov et al. 2012; Men'shchikov 2013). In brief, getsources identifies structures over various scales through spatial decompositions at each wavelength and then combines the data to measure source properties based on information from all wavelengths. In this way, higher resolution information is used to identify structures at lower resolution. Moreover, getsources is designed to identify and extract intensity peaks over a varied background, and as such, the algorithm can be used on other bolometer instruments. Nevertheless, since the GISMO data are filtered, we restricted the spatial decompositions to $<2.5^{\prime}$. We selected 17 reliable detections based on conservative selection criteria: (1) signal-to-noise ratios $(\mathrm{S} / \mathrm{N})$ of $S / N>3$ in at least three of the Herschel+GISMO bands and (2) no flags indicative of unreliable detections, such as low $\mathrm{S} / \mathrm{N}$ or few detections in the multi-scale decompositions. A complete catalog of sources in Orion will be made available from the Herschel Gould Belt Survey.

Table 1 lists the 17 reliable detections. Nearly all sources were also observed at $1.3 \mathrm{~mm}$ by Chini et al. (1997) and are labeled following their naming scheme. For those sources that were not observed by Chini et al. (1997), we used the 
Table 1. Source properties.

\begin{tabular}{lccccccccc}
\hline \hline & & & \multicolumn{4}{c}{ Dust temperatures } & \multicolumn{3}{c}{ Gas temperatures $^{c}$} \\
Name $^{a}$ & HOPS $^{b}$ & RA & Dec & $T_{\text {dust }}$ & $\beta$ & $M$ & $T_{\text {gas }}$ & $\beta$ & $M^{\prime}$ \\
& & $(\mathrm{J} 2000)$ & $(\mathrm{J} 2000)$ & $(\mathrm{K})$ & & $\left(M_{\odot}\right)$ & $(\mathrm{K})$ & & $\left(M_{\odot}\right)$ \\
\hline NW167 & 096 & $5: 35: 30.0$ & $-4: 58: 48$ & $17.7 \pm 2.2$ & $1.8 \pm 0.2$ & $0.8 \pm 0.3$ & $\ldots$ & $\ldots$ & $\ldots$ \\
NW165 & 383 & $5: 35: 30.0$ & $-4: 59: 46$ & $20.5 \pm 2.5$ & $1.8 \pm 0.1$ & $0.2 \pm 0.1$ & $\ldots$ & $\ldots$ & $\ldots$ \\
MMS 2 & 092 & $5: 35: 18.6$ & $-5: 00: 31$ & $14.6 \pm 1.1$ & $2.0 \pm 0.1$ & $4.4 \pm 1.4$ & 16 & $1.9 \pm 0.1$ & $3.2 \pm 0.2$ \\
MMS 4 & $089^{d}$ & $5: 35: 20.4$ & $-5: 00: 51$ & $9.7 \pm 0.6$ & $2.9 \pm 0.2$ & $14 \pm 0.1$ & 14 & $2.0 \pm 0.1$ & $2.4 \pm 0.1$ \\
MMS 5 & 088 & $5: 35: 22.6$ & $-5: 01: 14$ & $21.5 \pm 2.5$ & $1.5 \pm 0.1$ & $0.6 \pm 0.2$ & 19 & $1.7 \pm 0.1$ & $1.0 \pm 0.1$ \\
MMS 6 & 086 & $5: 35: 23.6$ & $-5: 01: 31$ & $24.9 \pm 3.3$ & $1.3 \pm 0.1$ & $0.8 \pm 0.3$ & 27 & $1.2 \pm 0.1$ & $0.7 \pm 0.1$ \\
MMS 7 & 084 & $5: 35: 26.6$ & $-5: 03: 57$ & $28.0 \pm 4.4$ & $1.7 \pm 0.1$ & $0.3 \pm 0.1$ & 19 & $2.2 \pm 0.1$ & $0.9 \pm 0.1$ \\
MMS 8 & $\ldots$ & $5: 35: 26.8$ & $-5: 05: 18$ & $12.3 \pm 0.8$ & $2.5 \pm 0.1$ & $3.5 \pm 1.0$ & 13 & $2.4 \pm 0.1$ & $2.8 \pm 0.2$ \\
MMS 9 & 078 & $5: 35: 26.3$ & $-5: 05: 45$ & $15.2 \pm 1.3$ & $2.1 \pm 0.1$ & $1.8 \pm 0.6$ & 19 & $1.8 \pm 0.1$ & $0.8 \pm 0.1$ \\
MMS 10 & $077^{d}$ & $5: 35: 32.4$ & $-5: 05: 49$ & $15.8 \pm 1.3$ & $2.1 \pm 0.1$ & $0.9 \pm 0.3$ & $\ldots$ & $\ldots$ & $\ldots$ \\
FIR 2 & 068 & $5: 35: 24.7$ & $-5: 08: 32$ & $14.2 \pm 2.0$ & $1.9 \pm 0.2$ & $0.8 \pm 0.4$ & 18 & $1.6 \pm 0.1$ & $0.4 \pm 0.1$ \\
FIR 3 & 370 & $5: 35: 27.8$ & $-5: 09: 34$ & $34.5 \pm 6.7$ & $1.7 \pm 0.1$ & $0.5 \pm 0.3$ & 28 & $1.9 \pm 0.1$ & $1.0 \pm 0.1$ \\
FIR 4 & 108 & $5: 35: 27.0$ & $-5: 09: 59$ & $28.5 \pm 4.3$ & $1.5 \pm 0.1$ & $2.1 \pm 0.8$ & 23 & $1.6 \pm 0.1$ & $3.8 \pm 0.2$ \\
FIR 6b & 060 & $5: 35: 23.6$ & $-5: 12: 04$ & $21.4 \pm 2.8$ & $1.9 \pm 0.2$ & $0.5 \pm 0.2$ & 17 & $2.2 \pm 0.1$ & $1.3 \pm 0.1$ \\
FIR 6a & $\ldots$ & $5: 35: 23.5$ & $-5: 12: 38$ & $17.0 \pm 1.6$ & $1.9 \pm 0.1$ & $2.8 \pm 1.0$ & 17 & $1.9 \pm 0.1$ & $3.0 \pm 0.2$ \\
FIR 6c & 059 & $5: 35: 21.7$ & $-5: 13: 14$ & $15.6 \pm 1.2$ & $2.1 \pm 0.1$ & $2.2 \pm 0.7$ & 24 & $1.6 \pm 0.1$ & $0.6 \pm 0.1$ \\
NW099 & 056 & $5: 35: 19.8$ & $-5: 15: 35$ & $23.3 \pm 3.2$ & $1.9 \pm 0.1$ & $0.5 \pm 0.2$ & 15 & $2.6 \pm 0.1$ & $2.2 \pm 0.1$ \\
\hline
\end{tabular}

Notes. ${ }^{(a)}$ Source names with "MMS" or "FIR" are from Chini et al. (1997) and source names with NW are from Nutter \& Ward-Thompson (2007). ${ }^{(b)}$ Source identifications from the Herschel Orion Protostar Survey (HOPS; Furlan et al. 2015). ${ }^{(c)}$ Results from SED-fitting with the dust temperature fixed to the kinetic gas temperature from Li et al. (2013). ${ }^{(d)}$ Tentative association with HOPS source.

source numbers from the $850 \mu \mathrm{m}$ SCUBA survey of Nutter \& Ward-Thompson (2007). Table 1 also lists the properties of our sources. We measured the source properties from fitting their SEDs using Eq. (1) with a mean molecular weight of $\mu=2.8$ in two ways: (1) fitting for the dust temperature, and (2) adopting a fixed dust temperature given by the $\mathrm{NH}_{3}$ kinetic gas temperature from Li et al. (2013). For dense objects $\left(\gtrsim 10^{6} \mathrm{~cm}^{-3}\right)$, the gas and dust are expected to be coupled, resulting in similar temperatures (e.g., Young et al. 2004; Ceccarelli et al. 2007). Several sources in Table 1, however, have gas and dust temperatures that differ by $>5 \mathrm{~K}$ and may be more tenuous. Alternatively, the gas and dust may not be tracing the same material (e.g., for protostellar objects, $\mathrm{NH}_{3}$ may trace the cool outer envelope, whereas the dust will trace primarily the warm inner envelope). The parameter errors correspond to $1 \sigma$ errors following a Monte Carlo analysis of the SED-fitting within the observational uncertainties.

We find a wider range in $\beta$ values $(1.2 \lesssim \beta \lesssim 3)$ for the cores compared to the $\beta$ values across the OMC $2 / 3$ filament from the unfiltered and filtered data, where $\beta$ was more uniform. Since large variations in $\beta$ suggest different dust properties, Table 1 suggests that the dense cores may have very different dust grain properties. Moreover, these different grain populations are very localized, as they are not noticeable in the analyses of the largerscale filament.

Table 1 shows MMS 6 has the lowest value of $\beta \sim 1.2-1.3$, whereas all remaining sources have $\beta>1.5$. These lower $\beta$ values for MMS 6 agree well with our previous results (see Figs. 4 and 5) and emphasize that this object may be unique. For the other sources in Table 1 , we find a wide range of $\beta$ indices and often large differences in $\beta$ from adopting either $T_{\text {dust }}$ and $T_{\text {gas }}$. Since most of our sources are protostellar (Megeath et al. 2012; Furlan et al. 2015), our single-temperature modified blackbody functions may be too simplistic to represent the source SEDs accurately. More extensive SED modeling with a better-sampled SED will improve our understanding of the dust properties.

\subsection{Evidence of dust grain growth?}

The dust emissivity index, $\beta$, is often used as an indirect probe of the dust grain population. Dust grains are most efficient at emitting radiation at wavelengths similar to their size, and as such, larger dust grains should emit more efficiently at long wavelengths than smaller dust grains. Nevertheless, $\beta$ is sensitive to many additional factors, including the dust composition, structure, and the presence or absence of ice mantles (e.g., Ossenkopf \& Henning 1994; Ormel et al. 2011). Moreover, depending on the physics and model, $\beta$ can remain relatively constant for different dust grain populations (Ormel et al. 2011).

Although the link between $\beta$ and dust grain populations is very complex, very low values of $\beta$ (e.g., $\beta \lesssim 1$ ) are most likely caused by substantial grain growth, whereas $\beta \simeq 2$ most likely reflects less processed dust associated with clouds and filaments (e.g., Testi et al. 2014). Thus, larger deviations from $\beta=2$ will still be informative even though we cannot connect small changes in $\beta$ unambiguously with grain evolution.

Using our unfiltered maps, we found $\beta \simeq 1.7$ throughout most of $\mathrm{OMC} 2 / 3$, with minimum values of $\beta \simeq 1.4$ toward MMS 6 (see Fig. 4). These values agreed well with the results from the filtered maps, where $\beta \simeq 1.6$ with lower values of $\beta \simeq 1.2$ toward MMS 6 . Thus, the MMS 6 source appears to be unique (see also Table 1). It is also one of the densest objects in OMC 2/3. Since Roy et al. (2013) found a strong link between column density and dust opacity in the southern parts of Orion A (excluding OMC 2/3), dust grain evolution in MMS 6 is expected. Alternatively, since MMS 6 is a protobinary system (Furlan et al. 2015), its lower $\beta$ values might be indicative of a more complicated SED than portrayed by our singletemperature modified blackbody functions (e.g., Shetty et al. 2009b). Therefore, while the lower $\beta$ indices in MMS 6 hint at unique dust grain properties, we cannot rule out a broadened SED due to multiple temperature components. 
Even if we assume that the lower $\beta$ indices toward MMS 6 are due to dust grain evolution, we find no evidence of $\beta<1$ either toward the sources or along the filament. In contrast, Schnee et al. (2014) found $\beta<1$ toward most of the OMC 2/3 filament on scales of $0.1 \mathrm{pc}$. Since our observations resolve this $0.1 \mathrm{pc}$ scale $\left(\sim 50^{\prime \prime}\right)$, these low values of $\beta$ should be detectable. Therefore, we find no evidence of the large dust grains in OMC $2 / 3$ suggested by Schnee et al. (2014). We discuss the possible reasons for this discrepancy in Sect. 4.3.

\subsection{Understanding the $\beta$ contradiction}

The results presented here and in Schnee et al. (2014) represent independent measurements of $\beta$ across OMC $2 / 3$ with different datasets and different techniques. Our two studies are inconsistent. Moreover, fixed values of $\beta=0.9$ with our SED-fitting analysis (see Sect. 3.1) yielded very poor results, with $\chi^{2}$ values that well exceeded the $\beta=1.3$ case (see Fig. 3). Indeed, fixed values of $\beta \lesssim 1.3$ yield subsequently inferior results that disagree with our observations.

Schnee et al. (2014) used gas temperatures from Li et al. (2013) to determine $\beta$ from their $1.2 \mathrm{~mm}$ and $3.3 \mathrm{~mm}$ observations, whereas we fitted our SEDs with a line-of-sight average dust temperature. If these temperatures were considerably different (e.g., due to tracing different material), they might explain the discrepancy in $\beta$. The gas and dust temperatures, however, showed good general agreement. For example, the gas temperatures were typically cooler than the dust temperatures by $\$ 5 \mathrm{~K}$, although some of the protostellar sources had larger variations of $\sim 10 \mathrm{~K}$ (see also Table 1). If we were to decrease all our dust temperatures to correspond better to the gas temperatures, $\beta$ would become steeper and deviate more strongly from the results in Schnee et al. (2014). Therefore, the kinetic gas temperatures cannot account for $\beta \sim 0.9$ in Schnee et al. (2014).

Alternatively, underestimated ratios of $1.2 \mathrm{~mm}$ to $3.3 \mathrm{~mm}$ emission would bias the results of Schnee et al. (2014) to lower $\beta$ indices. Figure 6 shows the SED for MMS 6 taking our Herschel+GISMO data and the $1.2 \mathrm{~mm}$ and $3.3 \mathrm{~mm}$ fluxes from Schnee et al. (2014). The best-fit SED (red curve) was determined from fitting the Herschel+GISMO data (see Table 1) and is thus determined independently of the $1.2 \mathrm{~mm}$ and $3.3 \mathrm{~mm}$ emission. The $1.2 \mathrm{~mm}$ MAMBO data show excellent agreement with our best-fit SED, whereas the $3.3 \mathrm{~mm}$ emission appears to be elevated by roughly a factor of two. Assuming that the $3.3 \mathrm{~mm}$ band was uniformly elevated by a factor of two across all of OMC $2 / 3$, ratios of $1.2 \mathrm{~mm}$ to "corrected" $3.3 \mathrm{~mm}$ emission would yield $\beta \simeq 1.5$. Such $\beta$ indices agree well with our results using the filtered Herschel and GISMO $2 \mathrm{~mm}$ data.

The $3.3 \mathrm{~mm}$ emission reported by Schnee et al. (2014) may have contributions from sources beyond dust, such as molecular line emission, anomalous microwave emission (AME), or freefree emission. Molecular line contamination in wide continuum bands is restricted mainly to very bright or very broad lines from CO gas (e.g., Drabek et al. 2012). For MUSTANG, these lines lie outside the bandpass (Schnee et al. 2014) and will not affect the continuum fluxes. AME is also expected to be negligible at $3.3 \mathrm{~mm}$. Schnee et al. (2014) used Planck observations to estimate the AME toward OMC 2/3 and found it to be well below the thermal dust emission (by over three orders of magnitude) at $\sim 3 \mathrm{~mm}$.

For free-free emission, Reipurth et al. (1999) found 14 sources of radio emission in the OMC 2/3 filament with the VLA in X-band $(3.6 \mathrm{~cm})$. Assuming that the radio emission traces optically thin free-free emission, Schnee et al. (2014)

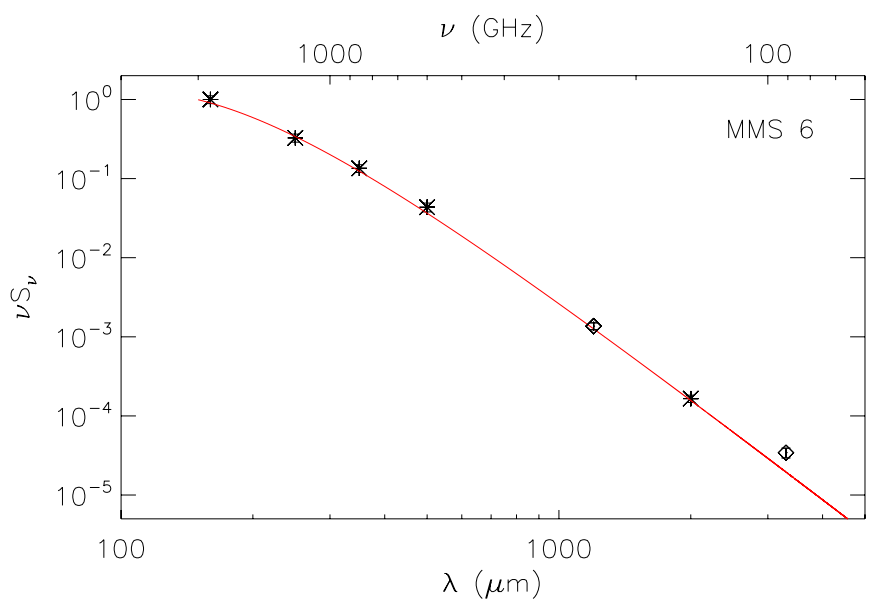

Fig. 6. Spectral energy distribution for MMS 6. Stars correspond to Herschel and GISMO $2 \mathrm{~mm}$ fluxes and diamonds show MAMBO $1.2 \mathrm{~mm}$ and MUSTANG $3.3 \mathrm{~mm}$ data from Schnee et al. (2014) for this source. The red curve shows the best-fit SED to the Herschel+GISMO data alone (see Table 1). Error bars show a 10\% flux uncertainty, representative of typical calibration errors.

determined that the contribution at $3.3 \mathrm{~mm}$ would be negligible. If the free-free emission traced at $3.6 \mathrm{~cm}$ is optically thick, however, it might contribute significantly to the $3.3 \mathrm{~mm}$ continuum. Nevertheless, such significant free-free contamination at $3.3 \mathrm{~mm}$ would similarly elevate the $2 \mathrm{~mm}$ emission by a factor of $\sim 1.2$, which would be noticeable compared to the $1.3 \mathrm{~mm}$ data in Fig. 6. Moreover, the radio sources in Reipurth et al. (1999) were generally compact $\left(\leqslant 8^{\prime \prime}\right.$ or $\left.\sim 0.02 \mathrm{pc}\right)$, making any contamination at $0.1 \mathrm{pc}$ scales unlikely. A more detailed analysis of the radio spectrum toward OMC $2 / 3$ is planned to characterize any free-free contributions to the observed millimeter fluxes toward the dense cores and along the filament.

If not from contamination, the elevated $3.3 \mathrm{~mm}$ observation could arise if the dust emissivity function deviates from a single power law. Many laboratory studies have found complicated dust emissivity functions at long wavelengths such that a single $\beta$ value does not describe well the efficiency of these grains (e.g., Coupeaud et al. 2011; Paradis et al. 2011). Meny et al. (2007) predicted shallower $\beta$ indices at long wavelengths $(>500 \mu \mathrm{m})$ that are due to changes in the structure of the dust grains themselves. Thus, the elevated fluxes at $3.3 \mathrm{~mm}$ might correspond to a more complicated dust emissivity function. Alternatively, the $3.3 \mathrm{~mm}$ data themselves might have systematic errors from the observations or the data reduction that have not been identified. Future observations at long wavelengths from larger cameras (e.g., MUSTANG-2; Dicker et al. 2014) will help address this concern.

\subsection{Comparison to Planck}

In Sect. 3.1, we recovered the large-scale emission at $2 \mathrm{~mm}$ using the Herschel data and different assumptions of $\beta$. Thus, the summation of our observed $2 \mathrm{~mm}$ data and the estimated largescale $2 \mathrm{~mm}$ emission should yield the total $2 \mathrm{~mm}$ emission of OMC 2/3. We compared such a total $2 \mathrm{~mm}$ map against similar observations from Planck $^{1}$ observations at $143 \mathrm{GHz}$ from the Full Release vs 2.0.

1 Planck is an ESA science mission with instruments and contributions directly funded by ESA Member States, NASA, and Canada. 
We extracted a $3^{\circ} \times 3^{\circ}$ map centered on OMC 1 from the Planck Legacy Archive. Since these data have a spatial resolution of $\sim 7.3^{\prime}(\sim 1 \mathrm{pc}$ for Orion) at $143 \mathrm{GHz}$ (Meisner \& Finkbeiner 2015), we convolved our total $2 \mathrm{~mm}$ map (for $\beta=$ 1.7) to match Planck. (We note that the Planck spatial resolution corresponds to the width of our GISMO observations; see Fig. 1.) We converted both maps to units of $\mathrm{MJy} \mathrm{sr}^{-1}$ (see Planck Collaboration IX 2015, for the Planck conversion) and placed all maps onto a common grid. Compared to the Planck $143 \mathrm{GHz}$ data, our total $2 \mathrm{~mm}$ map underestimated the $2 \mathrm{~mm}$ emission by only $\sim 8 \%$ on average (toward the inner $\sim 7^{\prime}$ of the filament), whereas the original (uncorrected) $2 \mathrm{~mm}$ map underestimated the $2 \mathrm{~mm}$ emission by $\sim 43 \%$ on average. Thus, our technique recovered the missing large-scale structure from the GISMO $2 \mathrm{~mm}$ data well.

We also compared our $\beta$ indices with those determined by Planck Collaboration X (2015). We used the $\beta$ maps from the Full Release vs. 2.0 available in the Planck archive. These data have a resolution of $7.5^{\prime}$, similar to the width of the OMC $2 / 3$ filament. The Planck-derived $\beta$ map shows $1.6<\beta<2.0$ and a median of $\beta \simeq 1.7$, with higher $\beta$ values toward the north and south. Thus, the Planck-derived $\beta$ indices of OMC $2 / 3$ agree well with the results from our unfiltered maps.

With our SED-fitting technique, we can recover the largescale emission from ground-based data and determine $\beta$ at resolutions superior to those of Planck (e.g., 36" for our GISMO data versus $7.5^{\prime}$ for the Planck data at $2 \mathrm{~mm}$ ). Alternatively, we can use the approach taken by Csengeri et al. (2016), where the missing large-scale emission in ATLASGAL observations at $870 \mu \mathrm{m}$ was supplanted directly by Planck observations. At $870 \mu \mathrm{m}$, the Planck data have a resolution of $5^{\prime}$, which corresponds better to the missing large-scale emission in most ground-based facilities (e.g., see Fig. A.3).

\subsection{Temperature and column density}

Many studies have used SED fits to examine the temperature and mass structure of molecular clouds with fixed values of $\beta$. Here, we fitted SEDs with flexible values of $\beta$, which we expect to provide a more reliable measurement of the line-of-sight dust properties. Figure 7 shows the temperature maps from our unfiltered and filtered Herschel+GISMO analyses with corresponding $\mathrm{H}_{2}$ column density contours. We find the coolest temperatures $(<20 \mathrm{~K})$ generally toward the densest material (e.g., $N\left(\mathrm{H}_{2}\right)>2 \times 10^{22} \mathrm{~cm}^{-2}$ ) with the filtered and unfiltered data agreeing within $\lesssim 2 \mathrm{~K}$ toward these regions.

Temperatures along the edges have $T \gtrsim 25 \mathrm{~K}$. At such warm temperatures, our SEDs are not fit reliably with $\lambda \geq 160 \mu \mathrm{m}$. Additional, short-wavelength data at $\$ 100 \mu \mathrm{m}$ are needed to constrain the fits better. Such an SED would also require more complicated models than our simplified modified blackbody functions to account for additional processes such as protostellar heating or non-equilibrium emission from very small dust grains (e.g., Schnee et al. 2008). Since such modeling is beyond the scope of this analysis, we instead excluded these warm regions.

In general, the dust temperatures appear to be most similar for the densest material from the unfiltered and filtered datasets. In contrast, their respective column densities show more significant variations, with lower values obtained from the filtered data than from the unfiltered data. For example, column densities of $>2 \times 10^{22} \mathrm{~cm}^{-2}$ cover an area of $\sim 30 \operatorname{arcmin}^{2}$ with the unfiltered data and only $\sim 5 \operatorname{arcmin}^{2}$ with the filtered data. Moreover, the peak column density decreases from $1.5 \times 10^{23} \mathrm{~cm}^{-2}$ to $1.0 \times 10^{23} \mathrm{~cm}^{-2}$ between the unfiltered and filtered results,

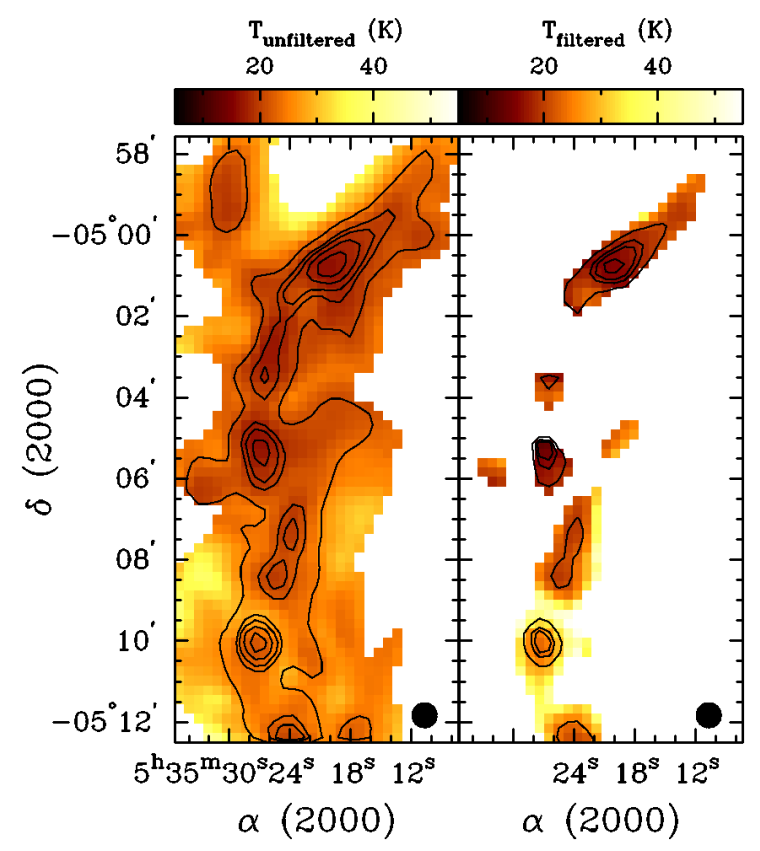

Fig. 7. Temperature maps of OMC $2 / 3$ using unfiltered data (left) and filtered data $($ right $)$. Contours show corresponding $\mathrm{H}_{2}$ column densities at $2,4.5,6,8.5$, and $12 \times 10^{22} \mathrm{~cm}^{-2}$. For the unfiltered maps, we show the results for $\beta$ as a free parameter.

respectively. These differences mean that we must be cautious when comparing column densities between studies with varying levels of spatial recovery.

Salji et al. (2015) used flux ratios of $450 \mu \mathrm{m}$ and $850 \mu \mathrm{m}$ data at $\sim 14$ " resolution from SCUBA-2 to determine temperatures and column densities for all of Orion A (including OMC 2/3) with the assumption of $\beta=2$. Comparing their results to ours from the filtered data, Salji et al. (2015) obtained cooler temperatures by $\sim 5 \mathrm{~K}$ and higher column densities by factors of several to an order of magnitude toward the densest material in the OMC $2 / 3$ filament. These differences may be due to the assumption of $\beta=2$. If we assume $\beta=2$ with the filtered data, the resulting temperatures decrease by $\sim 7 \mathrm{~K}$ and the column densities increase by several factors, both in better agreement with Salji et al. (2015). Since we found $\beta \simeq 1.6$ with the filtered data, assuming $\beta=2$ will have a significant effect on both temperature and column density (see Sect. 3.1).

For those regions with well-defined SEDs $(T<25 \mathrm{~K})$, we found a median temperature of $22 \mathrm{~K}$ and a median column density of $2.4 \times 10^{22} \mathrm{~cm}^{-2}$. These values agree well with the corresponding median values of $22 \mathrm{~K} / 2.4 \times 10^{22} \mathrm{~cm}^{-2}$ and $21 \mathrm{~K} / 2.6 \times 10^{22} \mathrm{~cm}^{-2}$ from Lombardi et al. (2014) and Stutz $\&$ Kainulainen (2015), respectively, even though both of these studies used Herschel data alone and fixed dust properties. Lombardi et al. (2014) adopted $\beta \simeq 1.8$ based on the Planckdetermined dust emissivities for Orion A, whereas Stutz \& Kainulainen (2015) adopted the dust opacities from Ossenkopf \& Henning (1994, OH5) that represent the expected dust grain properties for molecular clouds; we note that this particular dust opacity law has $\beta \simeq 1$. Since both studies used values of $\beta$ similar to our median value, it is unsurprising that our respective datasets match well. In general, our temperatures and column densities agree within $\sim 15 \%$ with those in both Lombardi et al. (2014) and Stutz \& Kainulainen (2015). Our analysis, however, was restricted to OMC $2 / 3$, and as such, we cannot comment on the results elsewhere in Orion A. 


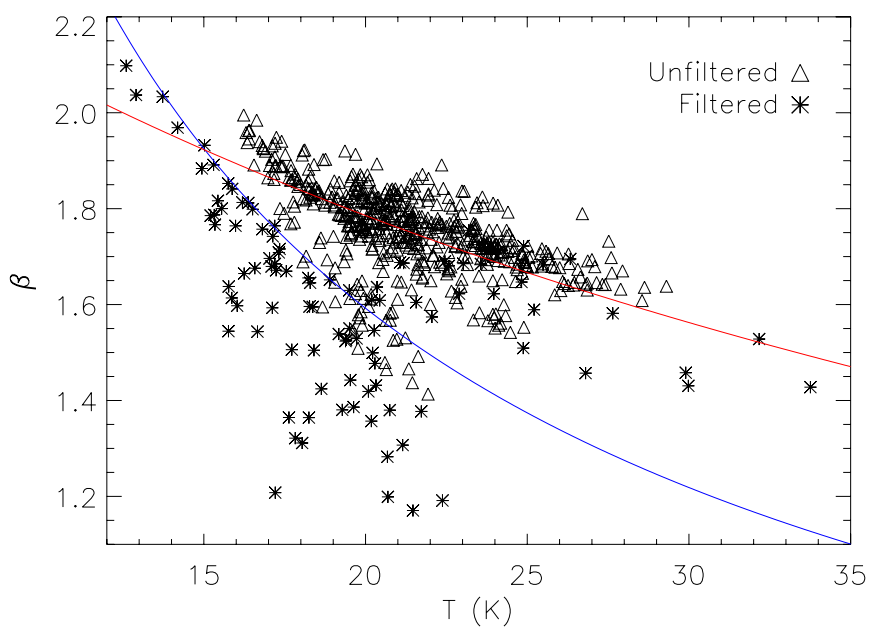

Fig. 8. Relationship between temperature and $\beta$ in OMC $2 / 3$. Triangles show the results from our unfiltered data and stars the results from the filtered data for pixels with $N\left(\mathrm{H}_{2}\right)>2 \times 10^{22} \mathrm{~cm}^{-2}$. The red curve shows the $T-\beta$ relation from Dupac et al. (2003) and the blue curve shows the relation from Désert et al. (2008).

Figure 8 shows the temperature- $\beta$ relations as determined by our unfiltered and filtered Herschel+GISMO data. Both distributions show an anticorrelated relation as seen in other studies (e.g., Dupac et al. 2003; Désert et al. 2008; Planck Collaboration XXV 2011; Juvela et al. 2015), with a population of relatively warm $(\sim 20 \mathrm{~K})$ dust with low- $\beta$ values $(<1.6)$ that deviate from the main anticorrelation. These pixels are associated exclusively with the MMS 6 core.

Figure 8 includes $\beta$-temperature relationships from PRONAOS (Dupac et al. 2003) and Archeops (Désert et al. 2008) for comparison. Excluding the MMS 6 data, our unfiltered data agree better with the anticorrelation from Dupac et al. (2003), even though their data were restricted to $\lambda<600 \mu \mathrm{m}$. In contrast, the correlation from Désert et al. (2008) included $2 \mathrm{~mm}$ data, as in this study, but did not fit our observations. Désert et al. (2008), however, measured $\beta$ and temperature for individual clumps, whereas in the present study and in Dupac et al. (2003), $\beta$ and temperature were measured across an entire map, pixel by pixel. Thus, the $\beta$-temperature relation may differ between compact sources and the larger-scale diffuse cloud, which might reflect a temperature dependence in $\beta$ itself or changes in these properties that are due to dust grain evolution (e.g., Mennella et al. 1998; Boudet et al. 2005).

The anticorrelation in Fig. 8, however, might reflect the degeneracies between temperature and $\beta$ in the presence of noise (e.g., Shetty et al. 2009a; Juvela et al. 2013) or from temperature variations along the line of sight (e.g., Shetty et al. 2009b; Ysard et al. 2012). In particular, $\chi^{2}$ analyses (such as those presented in this study) have been shown to produce skewed $\beta$-temperature relationships whereas more thorough Bayesian analyses have reproduced models more reliably (Kelly et al. 2012; Juvela et al. $2013,2015)$. Nevertheless, the inclusion of long wavelength data can improve the reliability of SED fits via $\chi^{2}$ methods (Juvela et al. 2013), and indeed, our Herschel+GISMO data span a wide range in wavelengths, covering both the SED peak and the Rayleigh-Jeans tail.

We tested the ability of the Herschel+GISMO data to produce reliable measures of $\beta$ and temperature using model SEDs. We generated mock fluxes corresponding to emission with $T=$ $20 \mathrm{~K}$ and $\beta=1.7$, typical of what is seen in the OMC $2 / 3$ region. Then, we fitted the mock fluxes with modified blackbody functions assuming $10 \mathrm{~K} \leq T \leq 80 \mathrm{~K}$ and $0 \leq \beta \leq 5.0$,

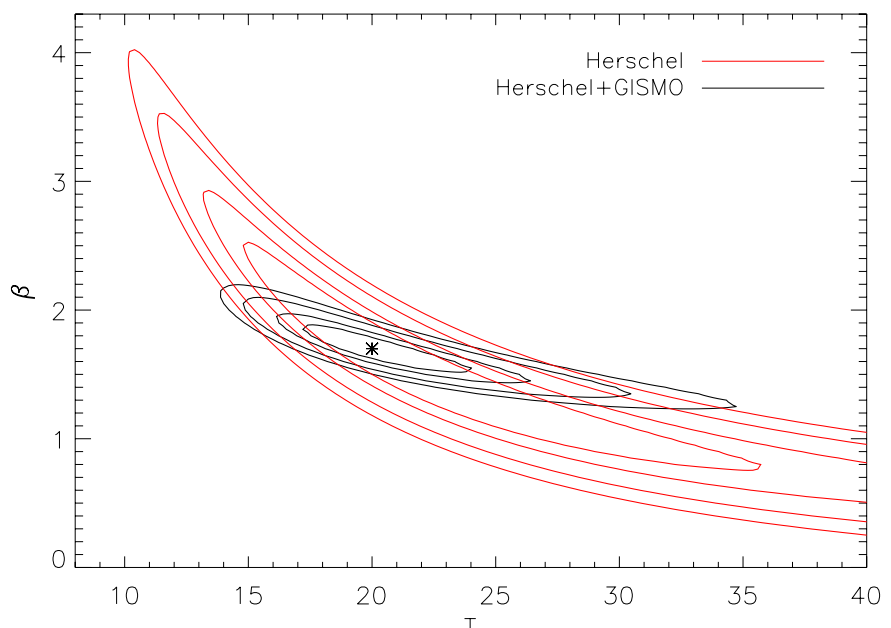

Fig. 9. Reduced $\chi^{2}$ contours from SED-fitting to Herschel $+2 \mathrm{~mm}$ data (black) and Herschel-only data (red) for mock data corresponding to $T=20 \mathrm{~K}$ and $\beta=1.7$. Contours correspond to reduced $\chi^{2}$ values of $\chi_{\text {red }}^{2}=0.5,1.0,2.0$, and 3.0, assuming four degrees of freedom for the Herschel $+2 \mathrm{~mm}$ data and three degrees for the Herschel-only data. The star shows the input temperature and $\beta$ values from our model.

assuming $10 \%$ calibration uncertainties in each band and the observed noise levels.

Figure 9 shows distributions of reduced $\chi^{2}$ produced by each temperature- $\beta$ pair based on SED fits using either the Herschel+GISMO bands and or the Herschel bands alone. Both distributions show curvatures that are indicative of the temperature- $\beta$ degeneracy from the calibration and noise uncertainties, although the Herschel+GISMO case does show much tighter constraints for both parameters. Additionally, the Herschel+GISMO case recovered the input $\beta$ and temperature values from our model, whereas the Herschel-only fits were skewed to warmer temperatures and lower $\beta$ indices. Even with larger uncertainties at $2 \mathrm{~mm}$ that represent the errors in our large-scale $2 \mathrm{~mm}$ offsets (see Sect. 3.1), we recovered the input model $\beta$ and temperature values with the Herschel+GISMO bands. Thus, the SEDs are well constrained by our Herschel and $2 \mathrm{~mm}$ data.

\subsection{Implications for future analyses}

Most previous studies that combined Herschel data with longer wavelength ground-based observations have been restricted to compact sources (e.g., Maury et al. 2011; Pezzuto et al. 2012; Stutz et al. 2013) or to filtering out the large-scale emission from the Herschel data (e.g., Sadavoy et al. 2013; Wang et al. 2015; Chen et al. 2015). Conversely, Stutz et al. (2010) combined Herschel and ground-based data for a Bok globule without applying any filtering or offsets. Adding a fixed offset (at $20 \%$ of the peak flux) to ground-based data did not affect their results, suggesting that the missing-large scale emission may not be significant for isolated Bok globules. Other studies have used Planck observations (Csengeri et al. 2016) or fixed offsets (Forbrich et al. 2015) to recover the large-scale emission from ground-based data and characterize the dust properties in clouds.

Using the technique outlined in Sect. 3.1, we can combine ground-based data with Herschel data without filtering or assuming a fixed offset. Our technique, however, is best used with ground-based observations at wavelengths far on the RayleighJeans tail of the SED. For example, earlier tests with SCUBA-2 data at $850 \mu \mathrm{m}$ were unable to constrain the SED fits clearly using a grid of offsets at $850 \mu \mathrm{m}$ (Sadavoy et al. 2013). Since our 
GISMO $2 \mathrm{~mm}$ data are farther along the Rayleigh-Jeans tail, we have better leverage to constrain $\beta$ and the large-scale structure.

\section{Conclusions}

We have measured the dust emissivity index, $\beta$, across the high-mass star-forming OMC 2/3 filament using Herschel 160-500 $\mu \mathrm{m}$ and GISMO $2 \mathrm{~mm}$ observations and several methods to combine these datasets. In particular, we produced the most complete and robust $\beta$ map of OMC $2 / 3$ at $36^{\prime \prime}$ $(\sim 15000 \mathrm{AU})$ resolution. Our main conclusions are listed below.

1. Using a technique that was first proposed in Sadavoy et al. (2013), we determined $\beta$ and recovered the missing large-scale emission from the $2 \mathrm{~mm}$ data simultaneously. We tested our technique against filtering the large-scale emission from the Herschel data and examining individual compact sources themselves. We found that our technique is consistent with these additional analyses and also with observations from Planck.

2. We found that $\beta>1.2$ everywhere in OMC $2 / 3$, with most of the filament having $\beta=1.7-1.8$. The lowest $\beta$ indices were consistently associated with the MMS 6 core, which may indicate unique dust grain properties toward this object. Nevertheless, the SEDs associated with MMS 6 are complicated by it being a protobinary system, and the lower $\beta$ values might reflect imperfect SED fits based on single-temperature modified blackbody functions.

3. We did not reproduce the very low indices of $\beta \simeq 0.9$ found by Schnee et al. (2014). Instead, we found that their $3.3 \mathrm{~mm}$ observations appear to be elevated by roughly a factor of 2, which biased their measured $\beta$ indices to such low values. These elevated fluxes may indicate significant contamination in this band or deviations from a single power law in the opacity curve. At this time, we cannot determine the cause.

4. The $2 \mathrm{~mm}$ observations provided a strong constraint for the SED fits, resulting in relatively reliable measurements of $\beta$ and temperature over the Herschel data alone. Like many other studies, we found an anticorrelation between temperature and $\beta$.

We determined $\beta$ across the OMC 2/3 filament without filtering the Herschel data or assuming a diffuse background offset value. Our technique is reliable and easily applicable to other clouds observed with Herschel and similarly long-wavelength, groundbased facilities. Future analyses of dust properties in molecular clouds can use this method in lieu of removing the large-scale emission that made Herschel submillimeter data so unique.

Acknowledgements. We thank the anonymous referee for providing helpful comments and suggestions. This work was possible with funding from the Natural Sciences and Engineering Research Council of Canada PDF award. The authors thank I. Hermelo, A. Kovács, and J. Staguhn for valuable assistance with the GISMO observations and data reduction, and T. Stanke for the MAMBO observations of OMC 2/3. IRAM is supported by INSU/CNRS (France), MPG (Germany) and IGN (Spain). This project used data from the Herschel Science Archive and the Planck Legacy Archive.

\section{References}

André, P., Men'shchikov, A., Bontemps, S., et al. 2010, A\&A, 518, L102 Beckwith, S. V. W., \& Sargent, A. I. 1991, ApJ, 381, 250
Bernard, J.-P., Paradis, D., Marshall, D. J., et al. 2010, A\&A, 518, L88 Boudet, N., Mutschke, H., Nayral, C., et al. 2005, ApJ, 633, 272

Ceccarelli, C., Caselli, P., Herbst, E., Tielens, A. G. G. M., \& Caux, E. 2007, in Protostars and Planets V, eds. B. Reipurth, D. Jewitt, \& K. Keil, 47 Chen, M. C.-Y., Di Francesco, J., Johnstone, D., et al. 2015, ApJ, submitted Chini, R., Reipurth, B., Ward-Thompson, D., et al. 1997, ApJ, 474, L135 Coupeaud, A., Demyk, K., Meny, C., et al. 2011, A\&A, 535, A124 Csengeri, T., Weiss, A., Wyrowski, F., et al. 2016, A\&A, 585, A104 Désert, F.-X., Macías-Pérez, J. F., Mayet, F., et al. 2008, A\&A, 481, 411

Di Francesco, J., Johnstone, D., Kirk, H., MacKenzie, T., \& Ledwosinska, E. 2008, ApJS, 175, 277

Dicker, S. R., Ade, P. A. R., Aguirre, J., et al. 2014, J. Low Temp. Phys., 176, 808

Drabek, E., Hatchell, J., Friberg, P., et al. 2012, MNRAS, 426, 23

Draine, B. T., \& Lee, H. M. 1984, ApJ, 285, 89

Dupac, X., Bernard, J.-P., Boudet, N., et al. 2003, A\&A, 404, L11

Enoch, M. L., Evans, II, N. J., Sargent, A. I., \& Glenn, J. 2009, ApJ, 692, 973

Forbrich, J., Lada, C. J., Lombardi, M., Román-Zúñiga, C., \& Alves, J. 2015, A\&A, 580, A114

Furlan, E., Fischer, W. J., Ali, B., et al. 2015, ApJ, submitted

Henning, T., Michel, B., \& Stognienko, R. 1995, Planet. Space Sci., 43, 1333

Johnstone, D., \& Bally, J. 1999, ApJ, 510, L49

Juvela, M., Montillaud, J., Ysard, N., \& Lunttila, T. 2013, A\&A, 556, A63

Juvela, M., Demyk, K., Doi, Y., et al. 2015, A\&A, 584, A94

Kelly, B. C., Shetty, R., Stutz, A. M., et al. 2012, ApJ, 752, 55

Kovács, A. 2008, in SPIE Conf. Ser., 7020, 1

Li, D., Kauffmann, J., Zhang, Q., \& Chen, W. 2013, ApJ, 768, L5

Lis, D. C., Serabyn, E., Keene, J., et al. 1998, ApJ, 509, 299

Lombardi, M., Bouy, H., Alves, J., \& Lada, C. J. 2014, A\&A, 566, A45

Maury, A. J., André, P., Men'shchikov, A., Könyves, V., \& Bontemps, S. 2011, A\&A, 535, A77

Megeath, S. T., Gutermuth, R., Muzerolle, J., et al. 2012, AJ, 144, 192

Meisner, A. M., \& Finkbeiner, D. P. 2015, ApJ, 798, 88

Mennella, V., Brucato, J. R., Colangeli, L., et al. 1998, ApJ, 496, 1058

Men'shchikov, A. 2013, A\&A, 560, A63

Men'shchikov, A., André, P., Didelon, P., et al. 2012, A\&A, 542, A81

Menten, K. M., Reid, M. J., Forbrich, J., \& Brunthaler, A. 2007, A\&A, 474, 515

Meny, C., Gromov, V., Boudet, N., et al. 2007, A\&A, 468, 171

Nutter, D., \& Ward-Thompson, D. 2007, MNRAS, 374, 1413

Ormel, C. W., Min, M., Tielens, A. G. G. M., Dominik, C., \& Paszun, D. 2011, A\&A, 532, A43

Ossenkopf, V., \& Henning, T. 1994, A\&A, 291, 943

Pagani, L., Steinacker, J., Bacmann, A., Stutz, A., \& Henning, T. 2010, Science, 329, 1622

Paradis, D., Bernard, J.-P., Mény, C., \& Gromov, V. 2011, A\&A, 534, A118

Peterson, D. E., \& Megeath, S. T. 2008, The Orion Molecular Cloud 2/3 and NGC 1977 Regions, Handbook of Star Forming Regions, Volume I: The Northern Sky, ASP Monograph Publications, 4, ed. Bo Reipurth, 590

Pezzuto, S., Elia, D., Schisano, E., et al. 2012, A\&A, 547, A54

Planck Collaboration XXIV. 2011, A\&A, 536, A24

Planck Collaboration XXV. 2011, A\&A, 536, A25

Planck Collaboration IX. 2015, A\&A, 571, A9

Planck Collaboration X. 2015, A\&A, submitted [arXiv: 1502 .01588]

Reipurth, B., Rodríguez, L. F., \& Chini, R. 1999, AJ, 118, 983

Roussel, H. 2013, PASP, 125, 1126

Roy, A., Martin, P. G., Polychroni, D., et al. 2013, ApJ, 763, 55

Sadavoy, S. I., Di Francesco, J., Johnstone, D., et al. 2013, ApJ, 767, 126

Salji, C. J., Richer, J. S., Buckle, J. V., et al. 2015, MNRAS, 449, 1782

Schnee, S., Li, J., Goodman, A. A., \& Sargent, A. I. 2008, ApJ, 684, 1228

Schnee, S., Mason, B., Di Francesco, J., et al. 2014, MNRAS, 444, 2303

Shetty, R., Kauffmann, J., Schnee, S., \& Goodman, A. A. 2009a, ApJ, 696, 676

Shetty, R., Kauffmann, J., Schnee, S., Goodman, A. A., \& Ercolano, B. 2009b, ApJ, 696, 2234

Shirley, Y. L., Huard, T. L., Pontoppidan, K. M., et al. 2011, ApJ, 728, 143

Staguhn, J., Allen, C., Benford, D., et al. 2008, J. Low Temp. Phys., 151, 709

Stutz, A. M., \& Gould, A. 2015, A\&A, submitted [arXiv: 1512.04944]

Stutz, A. M., \& Kainulainen, J. 2015, A\&A, 577, L6

Stutz, A., Launhardt, R., Linz, H., et al. 2010, A\&A, 518, L87

Stutz, A. M., Tobin, J. J., Stanke, T., et al. 2013, ApJ, 767, 36

Testi, L., Birnstiel, T., Ricci, L., et al. 2014, Protostars and Planets VI, 339

Wang, K., Testi, L., Ginsburg, A., et al. 2015, MNRAS, 450, 4043

Williams, J. P., \& Cieza, L. A. 2011, ARA\&A, 49, 67

Wright, C., Maddison, S., Wilner, D., et al. 2015, MNRAS, 453, 414

Young, K. E., Lee, J.-E., Evans, II, N. J., Goldsmith, P. F., \& Doty, S. D. 2004, ApJ, 614, 252

Ysard, N., Juvela, M., Demyk, K., et al. 2012, A\&A, 542, A21 


\section{Appendix A: Filtering the Herschel data}

Since GISMO is a ground-based instrument, its data are filtered on scales similar to the array footprint to remove the effects of a variable atmosphere. The Herschel data, however, are space-based and are not subject to the same level of filtering. In Sect. 3.2, we fitted SEDs to the Herschel+GISMO data using similarly filtered Herschel maps. We describe this filtering process in more detail here.

Following Wang et al. (2015), we removed the large-scale emission from the Herschel data by suppressing low spatial frequencies similar to a highpass filter. We determined which scales to suppress from the GISMO $2 \mathrm{~mm}$ data themselves to ensure that we filtered out similar spatial scales. In Fig. A.1, we show the GISMO $2 \mathrm{~mm}$ data at 36" resolution and the corresponding Fourier transformation. The bottom panel of Fig. A.1 shows the radial amplitude profile of the GISMO $2 \mathrm{~mm}$ frequency domain data. The radial profile was constructed from azimuthal averages in increasing bin sizes of one pixel. We fitted this amplitude profile with an exponential function, $f_{\exp }(r)$ (dashed curve). This exponential function represents the relative sensitivity to each scale. Therefore we used $1-f_{\exp }^{\text {norm }}(r)$ as our mask, where $f_{\exp }^{\text {norm }}(r)$ is normalized. Thus, at short uv radii, our mask reaches zero (e.g., filters out the large-scale emission), whereas our mask reaches one at large uv radii.

We multiplied our exponential mask with the Herschel data in frequency space, and then converted the products back to the image plane. Figure A.2 compares the unfiltered and filtered amplitude profiles for the Herschel bands following our technique. In general, we find that the filtered and unfiltered Herschel amplitude profiles agree well for spatial scales $\$ 2^{\prime}$, in line with the expectation that emission is recovered at levels of $\sim 90 \%$ at $\sim 2^{\prime}$. At larger spatial scales (e.g., $>5^{\prime}$ ), the amplitude decreases considerably because of filtering.

As an additional test of our method, we applied this Fourierspace filtering technique to the Herschel data of Perseus B1. These Herschel maps were filtered also through the SCUBA2 pipeline, which is expected to reflect the SCUBA-2 filtering process more reliably (see Sadavoy et al. 2013). Figure A.3 compares the Herschel amplitude profiles for the unfiltered observations, the Fourier-filtered data, and the SCUBA-2 pipeline filtered data. For the Fourier-filtered data, we used the original SCUBA-2 $850 \mu \mathrm{m}$ amplitude profile for B1 in a similar manner as described above for the GISMO data. The Fourier-filtering method generally removed more emission than the SCUBA-2 pipeline on scales $\gtrsim 5^{\prime}$. Nevertheless, both methods agree well for scales of $\$ 3^{\prime}$, where we expect most emission to be recovered. Thus, our Fourier-filtering technique appears to give a reasonable approximation of the filtering from ground-based facilities.

We note that a similar exercise to filter out emission corresponding to the MUSTANG pipeline (e.g., for comparison to Schnee et al. 2014) is inadvisable. The MUSTANG pipeline

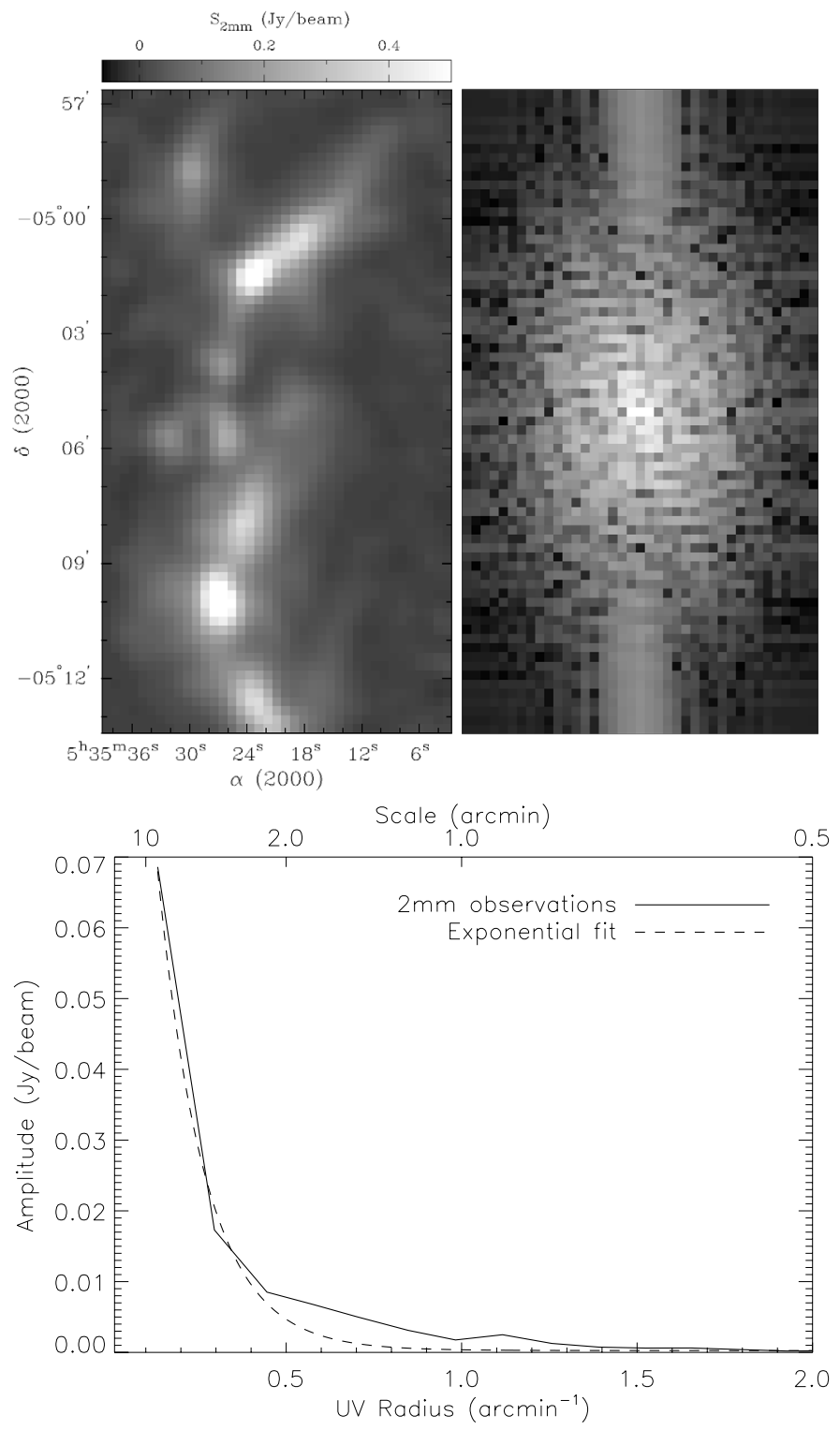

Fig. A.1. Top left: GISMO $2 \mathrm{~mm}$ observations of OMC $2 / 3$ at $36.3^{\prime \prime}$ resolution. Top right: Fourier transform of the convolved GISMO $2 \mathrm{~mm}$ data. Bottom: radial amplitude profile of the GISMO $2 \mathrm{~mm}$ data. The dashed line shows the best-fit exponential function to the data.

recovered $3.3 \mathrm{~mm}$ emission only to $\sim 1^{\prime}$. Since the SPIRE resolutions, particularly at $500 \mu \mathrm{m}$, are comparable to this largest recoverable scale, filtering the Herschel data with either the MUSTANG pipeline (e.g., as in Schnee et al. 2014) or with our Fourier transform method is highly suspect. In particular, the inherent resolution differences in the SPIRE data may result in a disproportionate fraction of emission being filtered. 


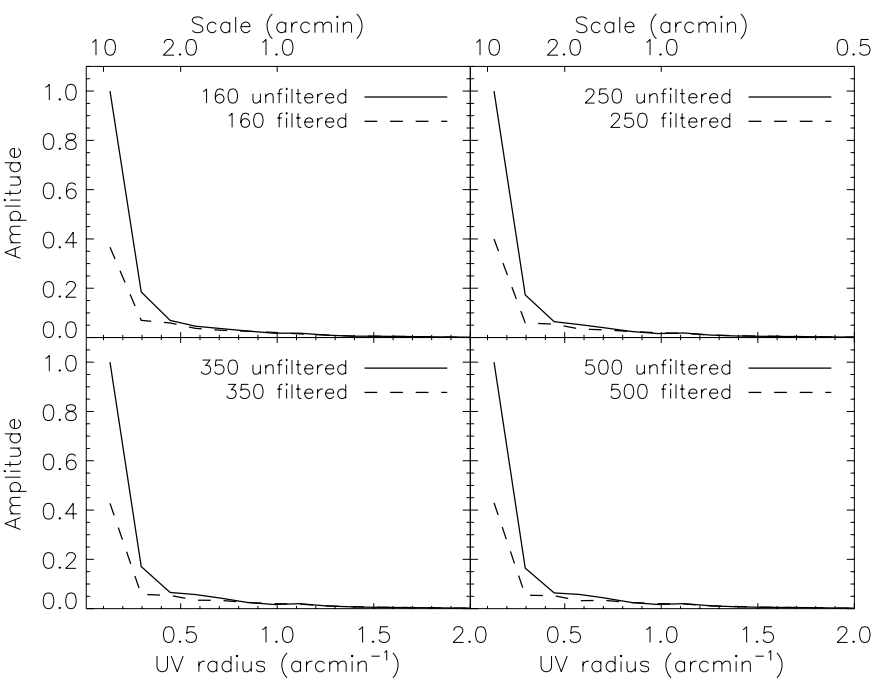

Fig. A.2. Radial amplitude profiles for the Herschel bands for a $32 \times$ 64 pixel region of OMC 2/3. The solid curves show the observed (unfiltered) amplitude profiles, normalized by the peak value. The dashed curves show the amplitude profiles of our filtered data following our large-scale masking. The filtered profiles were scaled by the same normalization factors as the unfiltered profiles. All data are relative to the $36^{\prime \prime}$ convolved Herschel data.

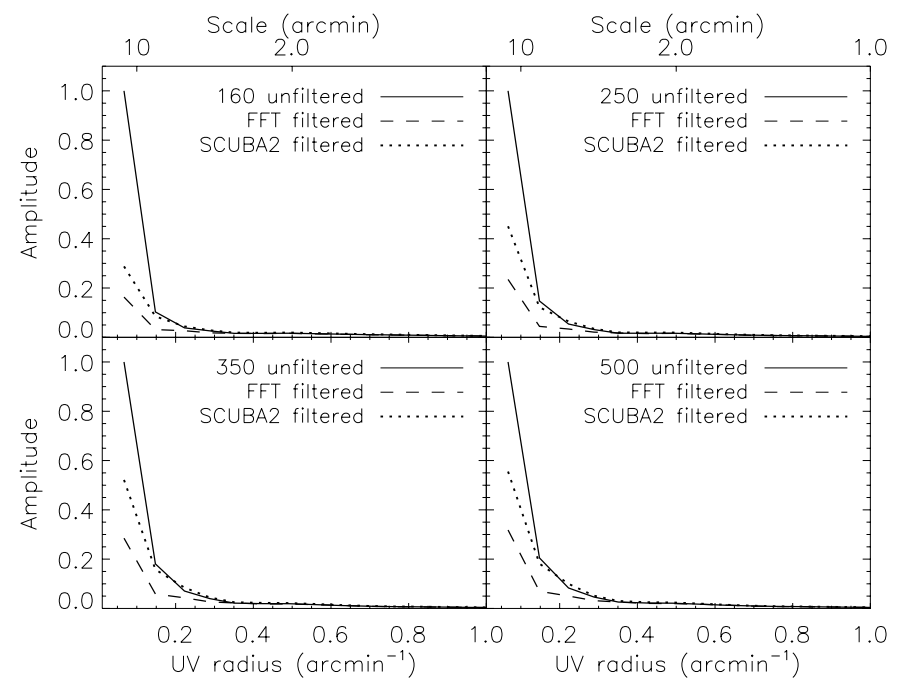

Fig. A.3. Same as Fig. A.2, but for a $64 \times 64$ pixel region of B1 in Perseus (Sadavoy et al. 2013). The dotted curves show the amplitude profiles for the corresponding Herschel maps that were filtered by the SCUBA-2 pipeline. 